a shorter time on fields 2 and 4 because for waves the time $(t)=3.815>1.96=t_{\alpha}$ and $(t)=3.252>1.96=t_{\alpha} ;$ and for wind $t=3.45>1.96=t_{\alpha}$ and $(t)=2.599>$ $1 \cdot 96=t_{\alpha}$ (Table 2).

During the voyages from the Gulf of Mexico to Europe, the ships on recommended routes also spent less time in fields 2 and 4 (stormy conditions) because for waves $(t)=2.00>1.96=t_{\alpha}$ and $(t)=3.053>1.96=t_{\alpha} ;$ and for wind $(t)=2.50>$ $1 \cdot 96=t_{\alpha}$ and $(t)=4 \cdot 167>1 \cdot 96=t_{\alpha}$ (Table 2).

More favourable conditions were also encountered by the ships on the recommended routes from Europe to the ports of USA and Canada and back (Table 3).

4. CONCLUSION. The hydro-meteorological conditions encountered point to the superiority of recommended routes over climatic ones. Taking advantage of the routes recommended by routing agencies, the ships sailing from Europe to North America reduced the number of days spent in the stormy area from 2 I per cent to 3.9 per cent of the voyage for waves and 29.2 per cent to ro per cent of the voyage for wind.

On the way back from North America to Europe the efficiency of the recommended routes was also considerable. The length of time spent in areas of wave height above $5 \mathrm{~m}$ was lowered from 20.5 per cent to 5 per cent and for wind from 39.5 per cent to 27.1 per cent of the whole voyage as compared with the climatic routes.

\title{
A World-wide Mechanization in Inertial Navigation Systems
}

\author{
G. Del Core and V. Nastro \\ (Istituto Universitario Navale, Naples)
}

An algorithm for inertial navigation by means of which the motion of the vehicle is referred to a developable surface tangential to the Earth is derived.

A first advantage is that the proposed scheme has no singularities at the pole. The algorithm is thus simpler than wander azimuth mechanization because the wander angle is a simple function of the longitude and the solution of six or more direction cosine differential equations is not necessary.

A further advantage is that the method allows the representation of the motion of the vehicle directly on a nautical chart.

It is well known that in inertial systems with gimballed platforms, the outputs of the horizontal accelerometers are employed to derive the ground velocity components of the vehicle in platform coordinates.

If the platform is locally level and north-pointing, east and north velocities $\left(V_{e}, V_{n}\right)$ are directly derived and easily converted into latitude-longitude coordinates $(\phi, \lambda)$ through dead-reckoning equations. Yet in high latitudes such a mechanization becomes difficult because of the convergence of the meridians; the azimuth rotation of the platform is a tangent function of latitude, and consequently as latitude increases such rotation approaches infinity.

This inconvenience is removed by varying the azimuth precession rate (wander 
azimuth mechanization); the axis of the platform will be at a variable angle $\alpha$ from north. It is necessary to calculate from time to time the angle $\alpha$ between the platform axis and the meridian.

In other mechanizations the platform axis remains pointed towards a fixed direction in the horizontal plane obtained through a rectangular grid relative to a reference meridian that will be chosen in the middle of the planned operations area. The platform always remains pointed parallel to the reference meridian in the north direction regardless of vehicle position if the time variation of the wander angle is rightly chosen.

Obviously the measured velocity components of the vehicle will no longer be $V_{n}, V_{e}$ but $V_{x}, V_{y}$ and the relationship between them is given by:

$$
\left.\begin{array}{l}
V_{n}=V_{y} \cos \alpha-V_{x} \sin \alpha \\
V_{e}=V_{x} \cos \alpha+V_{y} \cos \alpha
\end{array}\right\}
$$

where $\alpha$ is the wander angle between the geographical and the reference meridian.

It is also possible to refer the motion of the vehicle to a plane tangent to the Earth or to a developable surface that can be flattened to form a plane such as a cone or a cylinder.

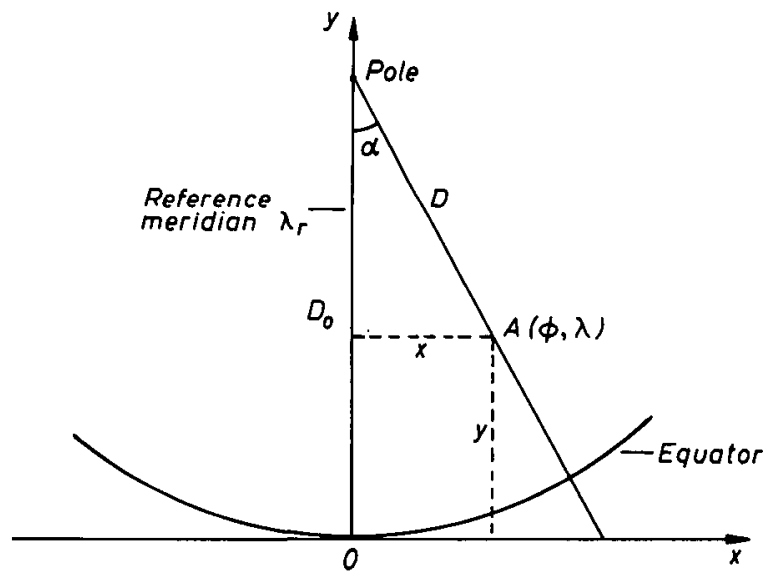

Fig. I

A bijection between geographical and caertesian coordinates of the plane can be established; remarkable advantages are attained if such relationships are the same as the fundamental orthomorphic nautical charts. Generally a mechanization can be derived from the mathematical relationships of a conical orthomorphic projection with one standard parallel referred to the middle of the operational area which interests our navigation. The latitude $\phi_{r}$ of such a point supplies the constant of the cone, while its longitude $\lambda_{r}$ identifies the reference meridian. The relationships in polar coordinates of such a chart are given by:

$$
\left.\begin{array}{l}
\alpha=k\left(\lambda-\lambda_{r}\right) \\
D=D_{0}\left[\tan \left(45^{\circ}-\frac{\phi}{2}\right)\right]^{k}
\end{array}\right\}
$$


where $k=\sin \phi_{\mathrm{r}}$ is the constant of the cone and $D_{0}$, the radius of the equator, is a known function of the standard parallel latitude:

$$
D_{0}=\frac{\cot \phi_{r}}{\tan \left[\left(45^{\circ}-\frac{\phi_{r}}{2}\right)\right]^{k}}
$$

Let us take a coordinate frame whose origin is at the point $\phi_{0}=0, \lambda_{0}=\lambda_{r}$, whose $x$ axis lies along the tangent at the equator and whose $y$ axis lies along the reference meridian (Fig. I). Equations (2) can thus be written in this form:

$$
\left.\begin{array}{l}
x=R D_{0}\left[\tan \left(45^{\circ}-\frac{\phi}{2}\right)\right]^{k} \sin k\left(\lambda-\lambda_{r}\right) \\
y=R D_{0}\left\{r-\left[\tan \left(45^{\circ}-\frac{\phi}{2}\right)\right]^{k} \cos k\left(\lambda-\lambda_{r}\right)\right\}
\end{array}\right\}
$$

where $R$ is the radius of the Earth.

Carrying the time derivates of (3) we obtain the $\dot{x}, \dot{y}$ components of the vehicle velocity on to cartographic representation after putting $\dot{\varphi}=V_{n} / R, \dot{\lambda}=V_{e} / R \cos \phi$ and $\dot{R}=V_{z}$ :

$$
\left.\begin{array}{l}
\dot{x}=\frac{k D}{\cos \phi} V_{x}+D \sin \alpha V_{z} \\
\dot{y}=\frac{k D}{\cos \phi} V_{y}+\left(D_{0}-D \cos \alpha\right) V_{z}
\end{array}\right\}
$$

Equations (4) may be integrated to give the instantaneous coordinates $x, y$ of the vehicle ; from them geographical coordinates can be deduced as follows:

$$
\left.\begin{array}{l}
\phi=90^{\circ}-2 \arctan \left[\frac{x^{2}+\left(y-R D_{0}\right)^{2}}{R^{2} D_{0}^{2}}\right]^{1 / 2 k} \\
\lambda=\lambda_{r}+\frac{1}{k} \arctan \left(\frac{x}{R D_{0}-y}\right)
\end{array}\right\}
$$

To maintain the platform axis parallel to the reference meridian requires an angular rate $\omega_{z}$ about the $z$ axis equal to $\sigma_{z}+\rho_{z}$, where $\sigma_{z}=\sigma \sin \phi(\sigma$ is the Earth angular rate) and $\rho_{z}$ is made up of two different components : (i) a first component, equal to $\lambda \sin \phi$, which accounts for the convergence of geographical meridians; (ii) a second component, $-\dot{\alpha}$, which counterbalances the rate of change of the wander angle $\alpha$; it is found by differentiating equation (2). Consequently:

$$
\rho_{z}=\frac{V_{e}}{R \cos \phi}\left(\sin \phi-\sin \phi_{r}\right)
$$

If the latitude $\phi_{r}$ of the reference point is zero, the cone becomes a cylinder and the mathematical relationships of the orthomorphic projection are the same as the Mercator chart:

$$
\left.\begin{array}{l}
x=R\left(\lambda-\lambda_{r}\right) \\
y=R \log _{\mathrm{e}} \tan \left(45^{\circ}+\frac{\phi}{2}\right)=R v
\end{array}\right\}
$$


The $\dot{x}, \dot{y}$ components of the vehicle velocity are equally derived from the differentiation of $(7)$ :

$$
\left.\begin{array}{l}
\dot{x}=\sec \phi V_{x}+\left(\lambda-\lambda_{r}\right) V_{z} \\
\dot{y}=\sec \phi V_{y}+v V_{z}
\end{array}\right\}
$$

By integrating (8) we obtain $x, y$, and from them geographical coordinates can easily be derived. The platform angular rate about the $z$ axis is derived from the (6) that, being $\phi_{r}=0$, becomes

$$
\rho_{z}=\frac{V_{e}}{R} \tan \phi
$$

The same results could be obtained from equations (4), replacing $k$ with the value zero; in fact we can easily prove that:

$$
\begin{aligned}
& \lim _{k \rightarrow 0} k D=1 \\
& \lim _{k \rightarrow 0} D \sin \alpha=\lambda-\lambda_{r} \\
& \lim _{k \rightarrow 0}\left(D_{0}-D \cos \alpha\right)=v
\end{aligned}
$$

If the reference point coincides with the pole $\left(\phi_{r}=90^{\circ}\right)$, the surface on which the motion of the vehicle is carried becomes a plane and consequently mathematical relationships of polar stereographic projection in polar coordinates are utilized as follows:

$$
\left.\begin{array}{l}
\alpha=\lambda-\lambda_{r} \\
D=2 R \tan \left(45^{\circ}-\frac{\phi}{2}\right)
\end{array}\right\}
$$

where, if we choose the same coordinate frame as in the conical projection, in cartesian coordinates they become:

$$
\left.\begin{array}{l}
x=2 R \tan \left(45^{\circ}-\frac{\phi}{2}\right) \sin \left(\lambda-\lambda_{r}\right) \\
y=2 R\left[1-\tan \left(45^{\circ}-\frac{\phi}{2}\right)\right] \cos \left(\lambda-\lambda_{r}\right)
\end{array}\right\}
$$

Carrying out the time derivatives and proceeding in the same way as for equation (4):

$$
\left.\begin{array}{l}
\dot{x}=\sec ^{2}\left(45^{\circ}-\frac{\phi}{2}\right) V_{x}+2 \tan \left(45^{\circ}-\frac{\phi}{2}\right) \sin \alpha V_{z} \\
\dot{y}=\sec ^{2}\left(45^{\circ}-\frac{\phi}{2}\right) V_{y}+2\left[1-\tan \left(45^{\circ}-\frac{\phi}{2}\right) \cos \alpha\right] V_{z}
\end{array}\right\}
$$


The same results could be found putting $k$ equal to unity in equation (4) and replacing $D_{0}$ with the value obtained from the limit:

$$
\lim _{k \rightarrow 1} D_{0}=2
$$

The angular rate of the platform about the $z$ axis becomes, by substituting $\phi_{r}=90^{\circ}$ in equation (6):

$$
\rho_{z}=\frac{-V_{e}}{R \cos \phi}(1-\sin \phi)
$$

that at the pole becomes the indeterminate form $\circ / \circ$; the value of $\rho_{z}$ at the pole is obtained from the limit of $\rho_{z}$ for $\phi \rightarrow 90^{\circ}$ and becomes zero. At the opposite pole, the angular rate becomes infinity and for this reason the mechanization is called 'unipolar'.

The above-mentioned algorithms are founded on the assumption that the Earth is a sphere; if one makes allowance for the ellipsoidal shape, mathematical relationships of conical maps for the spheroid can be utilized. Otherwise the geographical latitude $\phi$ must be converted into the geocentric latitude $\psi$ through the relation:

$$
\tan \psi=\left(1-e^{2}\right) \tan \phi
$$

where $e$ is the eccentricity. Moreover $R$ must be replaced by the equatorial radius $a$.

The mechanization we have been analysing is world-wide, and offers the advantage that the $x-y$ coordinates determined by the inertial navigator are directly compatible with any ortomorphic map for navigation if the reference point is constant (in practice a single reference point is sufficient).

In inertial systems with wander azimuth mechanization (as for LTN-51) the $z$ component of the platform angular rate with respect to an Earth fixed-coordinate system is constrained to zero; in such a system the latitude and the longitude of the vehicle and the time-varying wander angle $\alpha$ are computed from a matrix of direction cosines, and consequently at least six differential equations must be integrated to give the instantaneous values of the direction cosines.

In equation (6), if we put $\phi_{r}=\phi$ we obtain the same zero rate but the wander angle $\alpha$ will always be a simple function of the longitude as in equation (2). The reference latitude is continuously updated and adjusted to the actual latitude and the motion of the vehicle is referred to an osculating cone; the system will be analogous to the wander azimuth mechanization case $\left(\rho_{z}=0\right)$ but with a simpler scheme.

\section{R E F ER E N C ES}

' Ignagni, M. (1972). An all-earth inertial navigation scheme. Novigation (USA), 19, 3, Fall 1972.

${ }^{2}$ Nastro, V. (1978). Alcune soluzioni al problema del polo nella navigazione inerziale. Volume in Onore di Giuseppe Simeon. Naples: Giannini.

${ }^{3}$ Nastro, V. (1984). Navigazione Inerziale ed Integrata. Napoli : Luca Torre. 